TTR

Traduction, terminologie, re?daction

\title{
The Lonely Londoners en français : l'épreuve du métissage
}

\section{Hélène Buzelin}

Volume 13, numéro 2, 2e semestre 2000

Les Antilles en traduction

The Caribbean in Translation

URI : https://id.erudit.org/iderudit/037417ar

DOI : https://doi.org/10.7202/037417ar

Aller au sommaire du numéro

\section{Éditeur(s)}

Association canadienne de traductologie

ISSN

0835-8443 (imprimé)

1708-2188 (numérique)

Découvrir la revue

Citer cet article

Buzelin, H. (2000). The Lonely Londoners en français : l'épreuve du métissage. TTR, 13(2), 203-243. https://doi.org/10.7202/037417ar

\section{Résumé de l'article}

The Lonely Londoners en français : l'épreuve du métissage $\mathrm{E}$ En 1956, l'écrivain trinidadien Sam Selvon (1923-1994) publiait The Lonely Londoners, un roman rédigé intégralement dans une variété de langue jusque là stigmatisée et confinée au domaine dialogal : le vernaculaire créole trinidadien. Bien qu'il fasse aujourd'hui partie des « classiques » de la littérature anglo-caribéenne, ce roman n'a toujours pas été traduit en français. À partir d'une étude de la matérialité puis des fonctions textuelles de cette représentation, l'essai révèle que si The Lonely Londoners offre un exemple original de créolisation littéraire, sa traduction suppose quant à elle la représentation d'un français créolisé qui, en conflit apparent avec les normes du polysystème cible et les projets politico-linguistiques des élites franco-antillaises, n'a pas encore eu droit de cité dans le littéraire. Constatant l'existence d'équivalents micro-textuels aux marqueurs de créolisation présents dans l'original, l'auteure suggère que les enjeux sous-tendant la traduction d'un tel roman ne peuvent se définir en termes d'authenticité ou bien de choix entre une stratégie traductionnelle de résistance ou de transparence. En effet, s'inspirant à la fois des langues-cultures trinidadienne et britannique, The Lonely Londoners oblige à sortir des dichotomies traditionnelles et à penser la traduction comme un processus tripartite entre langues-cultures française-anglaise-antillaise. Loin de remplacer une dialectique étrangère (Angleterre-Caraïbes) par une dialectique domestique (France-Antilles), ou de remodeler l'étranger à traduire en fonction des termes du débat dans le contexte cible, la traduction vient en quelque sorte perturber ce débat et proposer de nouvelles avenues en matière de créolisation littéraire.
Tous droits réservés (C TTR: traduction, terminologie, rédaction — Les auteurs, 2000
Ce document est protégé par la loi sur le droit d'auteur. L'utilisation des services d'Érudit (y compris la reproduction) est assujettie à sa politique d'utilisation que vous pouvez consulter en ligne.

https://apropos.erudit.org/fr/usagers/politique-dutilisation/ 


\section{The Lonely Londoners en français : l'épreuve du métissage}

\section{Hélène Buzelin}

En 1950, Sam Selvon originaire de San Fernando (Trinidad) émigrait à Londres. Deux ans plus tard, il publiait un premier roman intitulé $A$ Brighter Sun pour lequel il reçut une bourse Guggenheim qui le conduisit à New York. C'est là-bas qu'il imagina le roman qui deviendrait, quelques années plus tard, The Lonely Londoners (1956); un récit en treize séquences dépeignant, avec humour, sur un mode inspiré du calypso, la vie et les affres d'un petit groupe d'émigrants londoniens d'origine principalement caribéenne, des personnages pour qui la quête d'un emploi, d'un toit ou d'un repas constituait un défi quotidien. Pour Selvon, The Lonely Londoners fut avant tout une expérience d'écriture originale, un processus en deux temps: après avoir tenté, en vain, durant deux mois, de rédiger ce roman en anglais "standard», l'auteur décida d'innover, d'adopter le vernaculaire comme langue de narration, de composer en somme l'intégralité de son récit dans ce qu'il désignerait plus tard comme un "modified Trinidadian dialect " (Selvon 1982). Aux yeux du critique et du lecteur anglophones, The Lonely Londoners représente un texte fondateur des littératures caribéennes'; un texte qui, cinquante ans après sa

\footnotetext{
${ }^{1}$ Le texte fut republié chez St. Martin's Press (New York) 1957; Mayflower Books (London) 1967; Longman (London) 1972; Longman collection Longman Caribbean Writers 1985, 6e impression 1998; TSAR (Toronto) 1991; Brown and Watson (London) 1967 sous le titre The Lonely Ones. Il fit l'objet d'une pièce radiophonique intitulée Eldorado West One diffusée sur BBC 4 (National Sound Archive T33201). Le scénariste londonien Biyi Bandele réalisa également une adaptation cinématographique commanditée par
} 
publication, continue à susciter au delà du champ des études postcoloniales, l'intérêt du public ${ }^{2}$. Pour le traducteur francophone, il constitue enfin un paradoxe et un défi : paradoxe puisque ce texte n'a toujours pas été traduit ${ }^{3}$... et donc défi pour celui ou celle qui entreprend de le faire.

Selvon n'est pas le premier à avoir inscrit dans le littéraire les parlers vernaculaires des Antilles anglophones. Bien avant lui certains avaient su exploiter ces variétés de langue ${ }^{4}$. Il n'est pas non plus le tout premier à avoir osé faire du vernaculaire la langue de narration. Sept ans plus tôt, Vic Reid avait ouvert la voie avec son roman historique New Day. L'originalité de Selvon réside donc moins dans sa décision de représenter le vernaculaire créole que dans la façon dont il l'a fait et dans les fonctions textuelles qu'il a assignées à ce vernaculaire.

Cette analyse part de deux constats, l'un relatif à la représentation littéraire du créole, l'autre à celle des sociolectes ou parlers « illégitimes" (Bourdieu 1982). Pour ce qui est de la première, plusieurs travaux ont révélé à quel point la créolisation textuelle passait

la BBC. L'ouvre de Selvon a fait l'objet de plusieurs thèses et monographies (Chukwu 1984; Luc-Cayol 1986; Nasta 1988, 1995; Wyke 1991; Clarke 1994; Dickison 1994; Looker 1996.

${ }^{2}$ Louise Doughty consacra récemment une édition de son émission $A$ Good Read à ce roman (BBC4 5/12/2000).

${ }^{3}$ Il existe une version en hollandais et une en italien. La seconde datant de 1997 est toujours disponible.

${ }^{4} \mathrm{~L}$ 'inscription du vernaculaire dans le roman anglo-caribéen s'amorça dès les $\mathrm{XVIII}^{\mathrm{e}}$ et XIXe siècles avec Kilner et Scott. Elle se poursuivit au début du $\mathrm{XX}^{\mathrm{e}}$ dans le cadre d'un courant réaliste social avec De Lisser, Mendes, Mittelholzer et Reid. Selon Ramchand (1970, p. 105), A Brigher Sun marque un tournant décisif car pour la première fois le vernaculaire y devint le véhicule de sentiments et de réflexions émanant d'un personnage « du peuple » dont les pensées constituaient la trame centrale du récit. Dans ce roman, le vernaculaire cessa d'être associé au comique ou à un réalisme social à visée contestataire et devint, d'après ce critique, " a language of consciousness ». The Lonely Londoners vint poser un nouveau jalon en introduisant ce langage dans la narration. La seconde moitié du siècle vit la publication d'un nombre croissant de romans écrits intégralement, ou plus souvent partiellement, en vernaculaire. 
par des stratégies de traduction du créole (emprunts, calques, transpositions, modulations ou adaptations) insérées dans le texte même ou dans le paratexte. Ces procédés se rencontrent dans les romans créolisés d'expression tant anglaise (Akai 1997; Bandia 1996; Lowry Weir 1982) que française (Bernabé 1992-1993; Jonassaint 1992-1993; DeSouza 1995; Hazaël-Massieux 1993, Confiant 2000) et ils constitueraient d'ailleurs, selon certains, l'une des caractéristiques des littératures post-coloniales (Ashcroft et al. 1989; Venuti 1998). Lane-Mercier (1995, p. 80) résume ainsi le second constat qui n'est pas propre aux littératures caribéennes : « [...] il est rare que le narrateur parle comme ses personnages, et ce à plus forte raison lorsque ses personnages "parlent mal" ". Autrement dit, les parlers illégitimes et $a$ fortiori les langues vernaculaires accèdent rarement à la narration'. Sur ces deux plans, The Lonely Londoners fait exception. Dans cet essai, je tenterai donc de montrer que ce roman représentant un exemple original de créolisation textuelle appelle des stratégies de traduction inusitées. Si elle s'apparente à une véritable " épreuve de l'étranger ", la traduction d'un tel texte offre donc, d'un autre côté, l'occasion d'explorer de nouvelles voies en matière de créolisation textuelle.

Faisant mienne la thèse de Lane-Mercier (1989) selon laquelle tout sociolecte ou dialecte romanesque résulte de la sélection d'un ensemble fini de marqueurs dont la nature et la distribution forment un système cohérent régi par des facteurs de lisibilité et une logique textuelle interne, $j$ 'analyserai la matérialité puis les fonctions textuelles du vernaculaire créole dans The Lonely Londoners avant de proposer, dans une troisième section, une possible démarche traductionnelle.

\section{Les procédés de créolisation linguistique : à la recherche du traducteur}

${ }^{3}$ Bien que les créoles anglais et français soient aujourd'hui considérés officiellement par les linguistes comme des langues à part entière, le stigma dont ils étaient chargés n'a pas entièrement disparu. En effet, les termes tels que broken English ou bad English communément utilisés pour désigner le créole (Winer 1993, p. 10; Roberts 1988, p. 14) reflètent le statut qui lui a longtemps été réservé. D'après Winer (p. 60), cette perception est encore courante chez les locuteurs créolophones eux-mêmes. L'enquête réalisée par Michelot (2000) suggère la persistance d'une attitude analogue dans les Antilles francophones. 
Quoique la créolisation textuelle passe autant par l'importation de genres ou de modes discursifs propres à la tradition orale — dans le cas présent il s'agit en particulier du calypso - que par celle d'une variété de langue, je m'en tiendrai ici à la dimension linguistique puisque c'est elle qui est susceptible de poser le plus de problèmes en traduction ${ }^{6}$. Le passage suivant tiré du roman donne un aperçu général de la nature et de l'agencement de ces marqueurs linguistiques.

It had a fellar call Five Past Twelve. A test look at him and say, 'Boy, you black like midnight.' Then the test take a second look and say, 'No, you more like Five Past Twelve.'

Five come from Barbados. During the war when Yankee was opening up base in Trinidad fellars was making a lot of many and the Five went to take part. One time he was taking out a Trinidad girl and the boys in the district didn't like it. They tell Five to leave the girl alone but Five ain't pay them no mind : the next night he was coasting with the girl around Queen's Park Savannah. The boys get a tin of pitch oil and throw on Five and start to run after him with a box of matches.

Five was a fellar, from the time he see you, he out to borrow money. You hardly have time to ask him what happening than he ask you if you could lend him ten shillings till Friday please God. So that all the boys start to take in front : from the time they see Five, they ask him to lend them two and six, that things really brown. (TLL, p. 94) ${ }^{7}$

\footnotetext{
${ }^{6}$ Genre trinidadien par excellence, le calypso remonte, d'après Warner (1982), au XVIII siècle, époque à laquelle immigrèrent des planteurs français et leurs esclaves. Il emprunte à diverses traditions orales telles que le picong, le belair, la ballade et le chant d'esclave et s'est surtout développé, après 1' Émancipation, dans le cadre d'une autre tradition populaire, celle du carnaval trinidadien. Dans sa forme actuelle, il présente les caractéristiques suivantes : « subversive irony, the melodramatic exaggeration of farcical anecdotes, racial stereotyping, repetition for dramatic effect and the inclusion of topical political material » (Nasta 1995, p. 86). Selon Fabre ([1979]1988, p. 215), ce genre s'apparenterait aussi au tall tale américain. Notons que Selvon s'inspire aussi d'une autre institution culturelle trinidadienne, le liming. Cette expression qui est devenue une icône culturelle trinidadienne renvoie à une pratique extrêmement populaire qui consiste à passer le temps avec des amis à fêter, discuter, ou simplement relaxer. Les personnages de Selvon "lime " souvent et le terme apparaît d'ailleurs à maintes reprises au sein du texte.
}

${ }^{7} T L L=$ The Lonely Londoners, TSAR (Toronto), 1991. 


\subsection{De la syntaxe et lexique}

Comme le suggère cet extrait, la langue du récit se démarque avant tout sur le plan de la morpho-syntaxe verbale. En effet, on retrouve dans ce texte les principales caractéristiques du système de prédication du créole anglais de Trinidad (cf. Solomon 1993) : absence de suffixe de temps ou de personne, possibilité d'omettre la copule devant un adjectif ou un complément de lieu (you black; things really brown), utilisation des particules does, did, et go pour indiquer le présent d'habitude, l'antériorité ou le futur lorsque nécessaire"; des formes telles que he must be do it et he had was pour exprimer la probabilité et l'obligation respectivement. Notons que dans ce système basé sur l'aspect plutôt que le temps, le contexte est indispensable à l'interprétation des relations temporelles: le temps " narratif » par défaut est le présent et non le passé. Par contre, les actions d'un récit amorcé par une particule telle que it had ou he was seront systématiquement interprétées comme relevant du passé, même si les verbes utilisés pour décrire ses actions sont non marqués. Ainsi dans l'amorce du roman : "One grim winter evening, when it had a kind of unrealness about London [...] Moses hop on a number 46 bus [...]", hop on sera interprété comme une action passée. À l'écrit, ce particularisme syntaxique produit à la fois un effet d'instantanéité similaire à celui du présent de narration et de fréquentes ambiguïtés, certaines actions pouvant parfois relever du passé ou du présent selon le point de référence adopté par le lecteur. Dans les syntagmes nominaux, l'omission quasi-systématique du morphème possessif (' $s$ ), l'emploi, moins systématique, de tournures du type she turn she back, sheself, l'absence occasionnelle de déterminants (opening up base), d'accord du nom en genre et en nombre ou de certaines prépositions sont également dérivés du créole. L'utilisation du pronom them comme adjectif démonstratif connote par contre un registre tant diastratique que dialectal. $\mathrm{Au}$ niveau des structures syntaxiques, The Lonely Londoners offre de nombreux exemples de fronting, procédé d'accentuation qui consiste à placer le verbe principal en début de

\footnotetext{
${ }^{8}$ Le système de prédication est avant tout basé sur l'opposition entre action finie et non finie, les formes non marquées étant interprétées comme finies. Ainsi, « they tell Five » correspond à un passé.
} 
phase : « in truth is that what happen to Henry » (TLL, p. 10); Selvon utilise de façon systématique la construction existentielle it have (it had a fellar call Five Past Twelve) héritée du créole à base lexicale française'; il tire également profit de l'absence de distinction formelle entre discours directs et indirects, propre au créole (de Trinidad), produisant des énoncés du type : " Galahad know what he want and he tell the fellar is all right, you go ahead, cut that jacket so and so, and dont forget I want a twenty-three bottom on the trousers » (p. 71), ou " the English people smiling isn't it a lovely say » (p. 86), ou encore " he tell him that he was sorry goodnight" (p. 89) etc. Adopté à l'échelle du roman, ce procédé de fusion syntaxique créole/anglais a pour effet de rendre l'inscription du créole à la fois omniprésente, puisque cette dernière s'affiche dans la construction de la moindre phrase, et relativement lisible pour le lecteur non-créolophone (cf. Ashcroft et al. 1989, p. 70; Mair 1990, p. 146).

Quoique moins nombreux, les marqueurs lexicaux sont également mobilisés. Outre les quelques termes évoquant la flore, l'alimentation ou les modes vestimentaires caribéennes: watchkong (espadrilles, apamols), calabash (calebasse), ochro (gombo), blackeyes peas (pois yeux-noirs), on retrouve plusieurs expressions telles que to kill oneself with laugh (mourir de rire), too bad (dans le sens de very much), a force ripe orange (orange cabanée), to cry big water (pleurer gros de l'eau), to laugh kiff kiff, to full up (pour to fill in) to thief, to dead, stupidness; quelques glissements sémantiques (fast, ignorant, a set of people). Plusieurs formules sont plus spécifiquement associées aux créoles de Trinidad, Tobago et Grenade : to make rab faire du grabuge), an oldtalk (vieux-parler, verbiage), a test (un type), a poorme-one (un crève-la-faim), my blood take you (je te trouve sympathique), to lime (drivailler, blaiser son corps), you best hads (you'd better) - idiomes dont certains proviennent du créole à base française, comme par exemple : it making hot/cold (il fait chaud/froid) calqué sur la structure française, tanty (fusion du français tante et de

'Bien que Trinidad n'ait jamais été sous domination française, de nombreux colons français s'y sont installés à la fin du XVIIJe siècle (Brereton 1981, pp. 13-31). Selon Winer : « FC [French Creole] remained widespread until the end of the $19^{\text {th }}$ century; in fact there were large numbers of more or less monolingual FC speakers in some areas well into the 1940s, and widespread bilingualism among its older speakers into the $1950 \mathrm{~s}-60 \mathrm{~s}$ » (1993, p. 9). 
l'anglais aunty), the vap tiré du français vapeur, grand-charge, dasheen (dachine), fete ou papa! ̇̀ ces termes s'ajoutent quantités d'expressions ou de lexèmes qui, loin d'être particulièrement trinidadiens, appartiennent à un registre plus diastratique que dialectal tels que spade (un noir, pej.), cuppa, char (une tasse de thé), a sport (une prostituée) etc. ${ }^{10}$

Il serait vain d'établir la liste des influences qui caractérisent le répertoire lexical de ce roman. Par contre, deux remarques s'imposent. Tout d'abord, comme le suggèrent ces exemples, les indices lexicaux proviennent principalement d'images (ex : to cry big water) ou de dérivations à partir de racines anglaises (ex : stupidness, to thief, to dead). Dans un cas comme dans l'autre, ils demeurent donc largement "transparents » pour le lecteur anglophone noncréolophone, les glissements sémantiques couverts (c-à-d. invisibles ) étant en outre assez rares. De plus, l'auteur a pris soin d'inscrire les créolismes lexicaux « opaques » dans des contextes explicites et/ou de façon si récurrente que les termes en deviennent peu à peu familiers. C'est le cas par exemple de coast a lime, oldtalk, spade ou test, qui jalonnent l'ensemble du récit. Cela ne signifie pas pour autant que soient définies, par le biais de périphrases explicatives, les expressions susceptibles de poser problème au lecteur non-créolophone. Au contraire, Selvon évite ici les stratégies de glossing (Ashcroft et al. 1989 , p. 61) qu'il avait utilisées dans son tout premier roman (Akai 1997, p. 188). Comme l'avait déjà remarqué Looker (1996, p. 74), The Lonely Londoners ne contient donc aucune définition ou traduction intratextuelle.

\subsection{Des marqueurs par défaut}

Si Selvon s'est inspiré de particularités syntaxiques et dans une moindre mesure lexicales, il est en revanche tout un pan de la langue qu'il n'a pas essayé d'inscrire dans son roman : la phonologie. Cette absence est d'autant plus surprenante que l'accent est d'habitude le premier aspect exploité par les auteurs tentant d'évoquer à l'écrit un parler associé à un sous-groupe régional ou social. Ici, la dimension

\footnotetext{
${ }^{10}$ Une étude exhaustive des marqueurs linguistiques dans l'œuvre de Selvon est fournie dans Wyke (1991). Hormis pour quelques termes relatifs à la flore, les traductions sont de moi et ne proviennent pas d'un dictionnaire bilingue. Pour une définition anglaise de ces termes, voir Ottley 1971 et Allsopp 1996.
} 
phonologique, a priori la plus apte à créer un effet de réel à peu de frais, est mise au rancart; concrètement, l'auteur n'utilise aucun eye dialect ${ }^{11}$. Seuls cinq ou six mots apparaissent sous une forme non standard - tess (pour test), fellar (pour fellow), ain't et Brit'n - une forme qui n'a d'ailleurs rien de particulièrement créole (Mair 1990, p. 145). Hormis ces quatre exceptions, Selvon ne recourt pas aux procédés courants tels l'accumulation d'apostrophes, l'ellipse, l'agglutination, la vocalisation des consonnes liquides ou une graphie plus ou moins phonétique évitant ainsi, selon moi, tant les connotations négatives que les problèmes de lisibilité que ces stratégies sont parfois susceptibles de générer. Si tout auteur possède son propre système de transcription, celui-ci est donc clairement motivé : ce n'est pas un accent mais une syntaxe et donc un rythme, un débit, des intonations que l'auteur nous donne à lire. Entreprise moins aisée, car il est plus facile d'imiter un accent qu'un contour mélodique, mais aussi moins propice à basculer dans l'ordre du stéréotype.

Le second aspect que Selvon et/ou son éditeur n'ont pas exploité est le paratexte. Alors que, d'après DeSouza (1995), les romans d'expression française un tant soit peu créolisés utilisent volontiers un ou plusieurs lieux para-textuels (notes de bas de pages, glossaires, lexiques renvoyant à des termes italicisés dans le texte, avertissements, voire annexes), autant d'espaces dans lesquels sont fournis des explications, traductions ou commentaires relatifs à la langue-culture caribéenne ${ }^{12}$, dans The Lonely Londoners, le récit se suffit à lui-même. Quel que soit le rôle de l'éditeur, il convient de mentionner que, tout comme l'absence d'eye dialect et l'inscription des lexèmes dans des contextes explicites, il s'agit là d'un choix également revendiqué par l'auteur (Selvon in Nasta et Rutherford 1995, p. 115; et Nasta 1988, pp. 78-79).

\footnotetext{
"Par eye dialect je désigne le recours à des modifications orthographiques sur des morphèmes lexicaux. Les traits morpho-syntaxiques analysés plus haut (absence de flexion désinentielle ou de cas possessif) n'entrent pas véritablement, selon moi, dans cette catégorie dans la mesure où ils ne touchent que les suffixes grammaticaux. En termes de lisibilité, leur impact est également différent.

12 Chronique des sept misères de Chamoiseau offre un exemple extrême d'utilisation du paratexte comprenant notes de bas de pages, chansons créoles en annexe, traductions de termes créoles.
} 


\subsection{La créolisation dans The Lonely Londoners ou "l'invisibilité du traducteur"}

Dans l'ensemble, le choix des marqueurs reflète un souci évident de donner à lire un texte qui tout en s'affichant à chaque ligne, dans chaque phrase, comme créolisé soit également des plus « fluides》: l'orthographe n'est jamais phonétique; si les structures syntaxiques sont assurément dérivées du créole, il n'est toutefois aucune phrase qui ne soit incompréhensible pour le lecteur non-créolophone; les emprunts lexicaux se limitent à quelques mots clés dont la répétition au fil du roman permet de saisir précisément le sens. En bref, comme le suggérait un certain Maurice Richardson qui recensa le roman lors de sa première parution " his syntax has an immediate readability of its own » (1956, p. 846) et le romancier n'a donc nul besoin d'ajouter des gloses ou des traductions créole/anglais.

$\mathrm{Si}$, comme le soutient Akai (1997), le roman anglo-caribéen est une traduction au sens culturel et linguistique du terme, dans le cas de The Lonely Londoners cette traduction semble tout au plus sousjacente et ne s'affiche en aucune manière de façon ouverte. En effet, loin d'être détaché ou mis en relief, le créole se fond ici à l'ensemble du texte. En cela, The Lonely Londoners correspondrait à ce que DeSouza considère comme le troisième et dernier stade de créolisation textuelle: " la fin de l'enfermement du créole dans un carcan de guillemets ou d'italiques [ $1^{\text {er }}$ stade], l'abolition des notes explicatives [2 $2^{\text {eme }}$ stade] " (1995, p. 186) ou, pour transposer la distinction linguistique de Chaudenson $(1979$, p. 135) dans le littéraire, le passage d'un discours "mixte " (diglottique) à un discours " métissé ». Notons enfin que ce narrateur qui refuse de jouer les traducteurs n'agrémente pas non plus son discours de gloses ou de précisions didactiques. En fait, ses rares commentaires linguistiques sont moins explicatifs qu'ironiques :

One time Moses meet Harris by Queensway buying daffodil from a barrow boy.

'Ah, you going in for horticulture now,' Moses tone.

The old Harris smile. 'No, I'm going to have high tea with Lord -'s daughter, and I thought it would be a nice gesture to take some flowers along'. 
Man, when Harris start to spout English for you, you realise that you don't really know the language. (TLL, p. 95)

\section{Et il en va de même des références culturelles :}

Now Moses don't know a damn thing about Jamaica - Moses come from Trinidad, which is a thousand miles from Jamaica, but the English people believe that everybody who come from the West Indies come from Jamaica. (TLL, p. 12)

En somme, dans The Lonely Londoners, les traductions interlinguistiques explicites sont absentes et les gloses, faussement didactiques, semblent avant tout le véhicule d'une douce ironie. Paradoxalement, ce roman dans lequel ont disparu toutes traces de traducteur et de traduction rappelle à quel point, loin d'être une simple nécessité ou, comme le suggère Akai (1997), une démarche allant pour ainsi dire de soi, la traduction intra- ou para-textuelle du créole vers l'anglais/français constitue aussi une stratégie délibérée dont l'utilisation relève autant d'une visée proprement textuelle que des contraintes qui s'imposent à l'auteur. Comme le souligne DeSouza, "l'emploi de notes explicatives incises en bas de page ou dans un glossaire ", en somme toutes les stratégies de traduction intra-textuelles ou para-textuelles "persiste[nt] à indiquer le caractère "autre" du créole " $(1995$, p. 179). En ayant représenté le vernaculaire à l'échelle du roman sans pour autant faire endosser au narrateur le rôle de l'interprète-traducteur, en adoptant un discours plus métissé que mixte, Selvon a au contraire choisi de faire du créole la norme, de donner pour ainsi dire au lecteur l'impression qu'un tel choix allait de soi. Par un minutieux travail d'adaptation et de réécriture, il établit un pont entre langues-cultures créole et anglaise (Fabre 1982) et offre surtout un exemple de créolisation textuelle avancée... avant l'heure. En effet, les techniques employées distinguent cet auteur de ses contemporains anglophones qui, plus souvent qu'autrement, utilisaient abondamment l'eye dialect, limitaient la représentation du créole aux composantes dialogales et n'hésitaient pas à mobiliser le paratexte ${ }^{13}$. Il semble que

13 Bien qu'il soit impossible d'être exhaustif, le constat s'applique aux principaux auteurs antérieurs à ou contemporains de Selvon: Edgar Mittelholzer, V.S. Naipaul, Claude McKay, George Lamming, Ismith Khan, Roger Mais, Wilson Harris, Merle Hodge, à l'exception, déjà mentionnée, de 
son approche diffère aussi de celle adoptée par les auteurs africains qui, d'après Bandia (1996), recourent plutôt à une stratégie de modification oụ de changement de code. Or ce faisant :

The native words and expressions are not particularly intrusive for readers. The author's European language of writing in which they are found does not often appear to have suffered any major violation of its lexico-grammatical structure. The reason for this is that the native words and expressions are usually used in such a way that they fit in with the syntactic and grammatical characteristics of the European language. (Bandia 1996, p. 144)

On ne pourrait imaginer procédure plus opposée puisque dans The Lonely Londoners la syntaxe anglaise est mise au contraire à rude épreuve. Depuis la "violation » des principales règles d'accord, pour reprendre les termes de Bandia, jusqu'à la suppression de certains éléments grammaticaux "clés", tels la copule ou le morphème possessif, en passant par le découpage parfois fantaisiste des unités du discours, ce roman nous permet de saisir toute la mesure de l'affirmation de Berman qui voyait dans la traduction «quelque chose de la violence du métissage " $(1984$, p. 16). Ainsi, Selvon remodèle-t-il litteralement la langue anglaise afin de satisfaire tant les critères de lisibilité que son projet expressif. Cela dit, comme on l'a laissé entendre plus haut, loin de se réduire à des contraintes extérieures, les questions de lisibilité s'intègrent elles aussi dans l'idéologie du texte. Dès lors, il convient de dépasser le niveau microtextuel, d'appréhender comment cette représentation s'organise au sein des différentes instances énonciatives du roman : les répliques actorielles et le discours narratif.

\section{Quelques fonctions narratives}

$\mathrm{Au}$ delà des fonctions réaliste et subversive maintes fois attestées (Barratt 1988; Fabre 1982, 1979; Looker 1996; Mair 1990; Nasta 1995; Ramchand 1988; Tabuteau 1993; Wyke 1991) quelle logique préside au choix et à la distribution des marqueurs de créolité présents dans The Lonely Londoners? En raison de sa structure anecdotique et de son affiliation à la tradition orale, ce texte a rarement été analysé

Vic Reid. Ainsi Merle Collins (1998, p. 90) rappelait récemment le travail de pionnier réalisé par Selvon. 
selon une approche narratologique. Pourtant, comme le souligne Kenneth Ramchand dans sa préface à la dernière édition du roman :

From looking at the narrator [and by inevitable extension, the narrating technique] we are drawn to recognise in Selvon's literary artefact a tightness of structure (the way the parts are put inevitably together); subtlety in the development and revelation of theme; linguistic cunning; and an appropriateness in the presentation and deployment of characters (Ramchand, in Selvon 1998, p. 10)

Dès lors, il convient d'analyser le rôle du dialecte littéraire recréé par Selvon en regard des macro-structures discursives et narratives de ce roman; saisir en quoi le choix et la distribution des marqueurs s'intègrent à l'idéologie textuelle d'ensemble.

\subsection{Les discours directs : du dialecte à l'idiolecte}

De prime abord, The Lonely Londoners se présente comme une succession d'anecdotes, de tableaux à travers lesquels sont dépeints des situations et personnages rappelant à bien des égards ceux du calypso: Moses, le " veteran Londoner » à qui l'on vient se confier ou demander conseil; le jeune Galahad récemment arrivé de Trinidad, beau parleur qui, par un curieux phénomène métabolique, a toujours chaud en hiver et froid en été; Cap le Nigérien paresseux, trickster par excellence qui survit au jour le jour en amadouant tout un chacun d'un sourire angélique; Tanty, la grand-mère jamaïcaine dont la parole ne finit jamais; Bart le Trinidadien au visage pâle tentant en vain de passer pour un Sud-américain ou bien Harris qui émule, de façon tout aussi vaine et stéréotypée, la langue et les manières de l'anglais. Ces Lonely Londoners sont au nombre de douze et, à l'exception de Harris, ils partagent tous globalement le même code. Les marqueurs sont certes légèrement plus nombreux dans les répliques de Tanty, l'aînée qui vient de quitter la Jamaïque, que dans celles de Cap, le Nigérien. Ceci dit, à l'échelle du roman, même la parole de Cap tend à s'aligner peu à peu sur celle des autres, évolution que le narrateur s'est d'ailleurs senti obligé de justifier : " At this stage of his acquaintance with the Boys he [Cap] does forget proper English and many times you would mistake him for a West Indian " (TLL, pp. 35-36). À l'opposé, toutes les répliques directes, isolées ou rapportées qui proviennent de personnages extérieurs au groupe sont en anglais « standard » ou, pour reprendre les termes du narrateur, en "proper English ». Le passage 
suivant illustre la façon dont Selvon distingue rigoureusement la voix des « Lonely Londoners » de celle des « autres » :

Like one time, when newspapers say that the West Indians think that the streets of London paved with gold a Jamaican fellar went to the income tax office to find out something and first thing the clerk tell him is, 'you people think the streets of London are paved with gold?' Newspaper and radio rule this country. (TLL, p. 8) (je souligne)

Les plus petits détails sont parfois les plus signifiants. Aussi, en ajoutant un simple auxiliaire, le narrateur parvient à se distancier bruyamment de la rumeur et, bien sûr, de celui qui la propage.

La répartition des marqueurs linguistiques dans les discours directs suggère sans détour la coexistence de deux mondes bien distincts : d'un côté celui des émigrants, de l'autre... tout le reste, sans d'ailleurs aucun souci de réalisme. Les rares personnages non émigrés qui font de brèves apparitions dans le roman partagent en effet le même code quel que soit leur niveau d'éducation, leur âge ou leur classe sociale : la petite amie anglaise de Galahad qui travaille à l'usine (TLL, p. 75) s'exprime de la même façon que le journaliste de l'Echo (TLL, p. 15), que le préposé du Employment exchange (TLL, p. 27), que le policier qui aide Tanty à trouver son chemin (TLL, p. 66) etc., une uniformisation qui, bien que découlant du caractère ponctuel et minimal du rôle de ces personnages, alimente en même temps l'idéologie de ce texte dont les dynamiques dialogales, les stratégies de focalisation, la macro-structure et finalement les propos du narrateur réaffirment constamment l'isolement de ces « Lonely Londoners » ${ }^{14}$.

${ }^{14}$ En effet, malgré la multitude d'acteurs, les dialogues entre les " lonely londoners » et les autres personnages obéissent toujours au même scénario qui en est un d'échec : quel que soit le contexte, on assiste ou bien à l'annulation de la parole d'autrui, à des cas d'incompréhension, au pire d'agression verbale. $\mathrm{Du}$ point de vue descriptif, l'auteur a adopté une stratégie de focalisation multiple par laquelle le lecteur accède à une vision de Londres partielle et fragmentée. Chaque personnage incarne littéralement un itinéraire au sens le plus concret, un parcours londonien spécifique avec lequel il s'est peu à peu familiarisé. Car, confie le narrateur, "London is a place like that. It divided up in little worlds, and you stay in the world you belong to and you don't know anything about what's happening in the other ones $[\ldots] »(T L L$, p. 58). 
Si ces émigrés partagent tous globalement un même code, leurs voix ne sont toutefois pas uniformes. En effet, toutes sont dotées de particularités idiolectales : depuis le "take it easy », expression fétiche d'un Moses désabusé et flegmatique, jusqu'à la dyslexie et la grossièreté de Big City - d'ailleurs censurée par le narrateur -, en passant par le fameux "oh lord" d'un Galahad toujours prêt à s'émerveiller et obsédé par les toponymes londoniens, les sempiternelles questions de Bart qui recherche désespérément sa fiancée à travers Londres ou bien les incessants commérages de Tanty, tous possèdent des idiotismes. Au delà de ces tics, on retrouve surtout à travers ces personnages et les situations dans lesquelles ils interagissent, la gamme des styles, des attitudes discursives identifiées par Winer (1993, p. 57) dans son analyse du créole trinidadien, de même que les nombreux termes servant à les décrire : talk English, talk big English pour celui qui, comme Harris cherche à impressionner de ses prouesses linguistiques; grandcharge pour celui qui, tel Galahad, cherche à intimider par la menace verbale; talk foolishness pour celui qui, comme Lewis, parle en l'air; to lime, lorsqu'on relaxe et l'on se laisse aller entre amis à des paroles légères, comme le font tous les dimanches matins les Lonely Londoners; to oldtalk lorsque l'on parle des choses d'antan et du pays lointain comme le font Ma et Tanty, le soir au coin du feu; ou bien sweet mouth lorsque l'on tente, comme Cap, de rouler l'autre par des paroles mielleuses. Cette diversité rappelle la place centrale qu'occupent la parole et les performances discursives dans l'existence de ces personnages. "If it was that we didn't get together now and then to talk about things back home, we would suffer like hell » (TLL, p. 114) confie Moses à Galahad, et le narrateur d'ajouter, à la toute fin «they only laughing, because they fraid to cry " (TLL, p. 125). Le propos est pour le moins clair et appuyé : s'il se distinguent des autres londoniens par leur parole, c'est aussi par le biais de cette parole, qu'il s'agisse d'anecdotes, de grands discours, de blagues ou de fabulations que ces émigrants largement ostracisés se construisent un sens du lieu (sense of place), qu'ils rompent leur isolement et tentent d'échapper, ne serait-ce que momentanément et métaphoriquement, à leur condition.

\subsection{Un narrateur caméléon}

Alors que dans les dialogues Selvon se plaît à superposer les codes afin d'amplifier leurs différences, dans la diégèse sa stratégie est tout autre, 
pour ne pas dire inverse. Bien que son identité demeure floue tout au long du roman, le narrateur adopte sans conteste la posture du "calypsonien" racontant ses anecdotes, mimant voire imitant ses personnages et interpellant son audience. Outre les interjections constantes au narrataire et l'accumulation de déictiques suggérant la présence physique d'un conteur: "So this is Galahad dressing up for the date " (TLL, p. 69); " so there Harris is [...]" (TLL, p. 96), il cumule les formules d'ancrage propres au récit oral. À défaut du canonique "once upon a time", les expressions " once " et "one time " inaugurent la moindre anecdote et donc, telles des amorces de strophes ou de couplets, structurent l'ensemble d'un chapitre. Ces répétitions rythment aussi, à plus petite échelle, les paragraphes dont certains s'apparentent à de véritables calypsos (Warner 1982, pp. 127129). Enfin, à l'image du calypsonien qui se caractérise par son habileté à « adopter les traits du personnage qu'il décrit » (Hill 1972, p. 64 , je traduis), ce narrateur qui partage globalement le même code que les émigrants n'hésite pas à moduler son discours en fonction de l'identité du personnage orientant le point de vue ou faisant l'objet du récit ou de la description.

Par exemple, dans la sixième séquence consacrée à la description du quartier de Harrow Road où réside la famille de Tanty puis au récit de la première escapade de ce personnage dans le centre de Londres, le narrateur ajuste peu à peu sa voix, la rend légèrement plus créolisée qu'à l'habitude et emprunte des expressions qui semblent tout droit sorties de la bouche de ce personnage, tel ce commentaire : " when she get off at the Prince of Wales she feel relieved. Now nobody could tell she that she ain't travel by bus and tube in London " (TLL, p. 67). Cette perméabilité du narrateur aux voix qui l'entourent fonctionne à l'échelle du roman avec les autres « lonely Londoners » (Thieme 1986) et en particulier avec le personnage de Moses. Elle repose sur une stratégie qui relève beaucoup plus de la légère modulation que du véritable changement de code et elle apparaît d'ordre plus idiolectal que dialectal. Ainsi, le lecteur attentif reconnaît de temps à autre tel ou tel tic langagier, il a l'impression d'entendre, dans la parole du narrateur, les intonations de Tanty, l'hypercorrection de Harris, mais il lui est bien impossible de déterminer le moment précis où cette modulation s'effectue. Ces brouillages énonciatifs sont accentués par le recours à des modes de réénoncation apparemment hybrides (Folkart 1991) qui relèvent du discours indirect d'un point de 
vue typographique et du discours direct d'un point de vue syntaxique. Ils sont enfin et surtout renforcés par l'usage récurrent d'un you polysémique qui traverse de part en part le roman et constitue, selon Maximin, " la véritable clé de voute de tout l'édifice » (Maximin 1996, pp. 362-363). Ces procédés sont particulièrement exploités à la fin du roman :

What happening, what happening man.

What the arse happening, lord ? What all of us doing, coasting lime, Galahad asking if anybody know the words of the song Maybe It's Because I'm a Londoner, Cap want two pounds borrow, Five only in town for the night and he want to know if he could sleep in Moses room, Big City coming tomorrow to full up the coupons (I nearly hit them last week), Lewis saying that Agnes come begging and if he should go to live with her again, Tolroy want to send Ma and Tanty back to Jamaica (them two old bitches, I don't know why they don't dead).

So what happening, Tolroy ? I don't see you with your guitar these days?

Every year he vowing to go back to Trinidad, but after the winter gone and birds sing and all the trees begin to put on leaves again, and flowers come and now and then the old sun shining, is as if life start all over again, as if it still have time, as if it still have another chance. I will wait until after the summer, the summer does really be hearts.

But it reach a stage, and he know it reach that stage, where he get so accustom to the pattern that he can't do anything about it. Sure, I could do something about it, he tell himself, but he never do anything. He used to wonder about back home, where he have a grandmother and a girl friend who always writing him and asking him why he don't come back, that they would go and live in Grenada, where her father have a big estate.

Why don't you go back.

What happening man, what happening.

If I give you this ballad ! Last night -

You went to see the Christmas tree in Trafalgar Square?

Harris giving a dance in Brixton next Saturday - you going ? (TLL, pp. 124-125) (je souligne)

À l'image de ce passage, l'ensemble de la séquence finale (pp. 121126) est basé sur le monologue intérieur du narrateur/personnage régulièrement entrecoupé de ces répliques décontextualisées et décousues desquelles surgissent les idiotismes de chacun. Par le biais de ces modulations idiolectales, de l'usage des déictiques et de 
l'absence de marqueurs typographiques, la distinction entre les paliers discursifs et narratifs s'obscurcit ${ }^{15}$.

Ces procédés ont pour effet de solidariser le narrateur des personnages l'entourant sans pour autant créer, du moins en surface, un discours uniforme et unique. Notons que ces ambiguïtés énonciatives se répercutant à l'autre pôle de la chaîne parlée modifient également la force illocutoire des énoncés proférés. En effet, tandis que les acteurs accèdent au palier narratif, leurs voix se fondant avec celle d'un narrateur qui s'efface peu à peu tout en tirant les ficelles, la distinction entre les différents you obsessionnels devient de plus en plus obscure : le you actoriel (par exemple de Moses à Galahad), le you de généralisation, le "you » à valeur de je (dans les monologues intérieurs de Moses), et enfin le you désignant le narrataire s'entremêlent indistinctement... le narrataire semble ainsi invité à se fondre avec l'acteur, à prendre place au sein de l'univers décrit, à se mettre littéralement à la place de ces « lonely Londoners ».

Tandis que se superposent les répliques décousues des " lonely Londoners ", la voix de Moses tend à se fondre avec celle du narrateur. D'après Ramchand (1998), cette accession du personnage principal au statut de narrateur constitue l'une des thématiques centrales du roman dont la structure temporelle s'avère dès lors beaucoup moins décousue qu'elle n'y paraissait à première vue. Reprenons du début, première séquence : un triste soir d'hiver, Moses se rend à Waterloo pour accueillir Sir Oliver Galahad venu de Trinidad pour tenter sa chance dans la capitale britannique. Dix ans plus tôt, Moses était lui aussi arrivé à Waterloo et, précise le narrateur, quand le jour viendrait, c'est de cette même gare qu'il repartirait. En attendant Galahad, Moses rencontre Tolroy, un Jamaïcain qu'il avait également accueilli, aidé, hébergé quelques années plus tôt, qui attend lui aussi le train dans lequel se trouve sa mère. Les années ont donc passé mais le rôle de Moses est demeuré le même. L'arrivée de Galahad sera l'élément déclencheur, le prétexte à une série de réminiscences. Ainsi, à travers le récit des premières années de Galahad dans la capitale, c'est en fait l'histoire de cet autre personnage qui sera indirectement racontée. Entre les trois ou quatre années qui séparent le point de départ (arrivée de Galahad) et la chute du récit, il s'opère un incessant

${ }^{15}$ Je m'inspire ici du modèle du récit encadré de Jaap Lintvelt (1978). 
chassé-croisé entre le passé de Moses et l'expérience présente de Galahad. Après l'arrivée (séquence 1), Galahad, guidé par Moses, découvrira l'Employment exchange (séquence 2). Dans les deux séquences suivantes, on abordera la question du logement par le biais d'une rétrospective de l'arrivée de Moses entrecoupée ça et là de répliques isolées entre Galahad et Moses. Après un détour par le quartier de Harrow Road (séquence 6), ce dialogue entre Moses et Galahad sera repris, de façon toujours elliptique (séquence 7): quelques mois ont passé, Galahad a trouvé un emploi et un logement. C'est l'été et il découvre à présent les plaisirs de la ville. Arpenter Hyde Park, donner rendez-vous à Charing Cross... Dans ce chapitre, Galahad se confie à Moses qui déclare revoir, à travers lui, ses premières années. On retrouvera à nouveau des bribes de dialogues dans les deux séquences suivantes (9-10) dont on sait tout au plus qu'elles se déroulent également en été. Pour les trois dernières séquences on retournera de nouveau en hiver: Galahad perd son emploi et son logement, il retourne chez Moses. Entre la première et la dernière discussion entre ces deux personnages, on aura donc assisté au parcours initiatique de Galahad et, à travers lui, à celui de Moses; on aura traversé trois ou quatre années, quatre années identiques les unes aux autres, sous le signe de la répétition ${ }^{16}$. Le système prédicatif représenté dans le roman, système dans lequel l'aspect prédomine sur le temps et où les ambiguïtés temporelles sont fréquentes, renforce jusqu'à un certain point l'économie narrative de ce récit dans lequel les saisons et les années s'enchaînent à un rythme effréné « [in] a great restless, swaying movement that leaving you standing in the same spot " (TLL, p. 125), où chaque année est identique à la précédente, où le seul changement est celui que procure l'alternance des saisons. L'accession du personnage au statut de narrateur et l'évocation du projet d'écriture de ce personnage viendront ouvrir une brèche à la toute fin du roman, suggérant la possibilité de rompre cette structure circulaire.

\subsection{Les moments narratifs : descriptions et anecdotes}

Outre l'identité des personnages avoisinants, les modulations de la voix narrative sont déclenchées par la nature du passage narratif. De façon

\footnotetext{
${ }^{16}$ Notons que la première scène de l'adaptation radiophonique diffusée par la $\mathrm{BBC}$ correspond à la dernière scène du roman.
} 
schématique, les récits (d'événements ou de paroles) de même que les descriptions de personnages sont volontiers contés dans un style créolisé et "oralisé ". Seules les descriptions de l'environnement extérieur, du paysage londonien, s'avèrent plus lyriques et plus " écrites ». Voici par exemple l'amorce du roman :

One grim winter evening, when it had a kind of unrealness about London, with a fog sleeping restlessly over the city and the light showing in the blur as if is not London at all but some strange place on another planet, Moses Aloetta hop on a number 46 bus [...]. (TLL, p. 7)

Si le tout début évoque une description de Londres digne d'un roman de Dickens, le créole s'immisce peu à peu et finit, quelques lignes plus bas, par prendre le dessus :

He wasn't in a good mood and the fog wasn't doing anything to help the situation. He had was to get up from a nice warm bed and dress and come out in this nasty weather to go and meet a fellar that he didn't even know. That was the hurtful part of it - is not as if this fellar is his brother or cousin or even friend; he don't know the man from Adam. (TLL, p. 7)

Il est possible d'extraire de ce roman certains passages particulièrement lyriques rédigés dans un anglais plus « standard » quoique toujours en partie créolisé :

In the grimness of the winter, with your hand plying space like a blind man's stick in the yellow fog, with ice on the ground and a coldness defying all effort to keep warm, the boys coming and going, working, eating, sleeping, going about the vast metropolis like veteran Londoners. (TLL, p. 122)

... et d'autres écrits dans un style plus dialectal (cf. infra pp. 2-3). Toutefois, là encore, ces variations sont subtiles et reposent sur des indices relativement discrets, tels les temps ou marques de ponctuation signalant un changement de rythme.

Ces différents types de modulation illustrent ce que lecteurs, romanciers et critiques semblent percevoir aujourd'hui comme l'une des principales réussites de Selvon : la capacité à retrouver une tonalité 
et l'ambiance d'une époque, «a tone and a mood » ${ }^{17} .$. Avoir capturé la musicalité du dialecte trinidadien, l'atmosphère, l'humeur mais aussi l'humour de ces personnages, tel serait le véritable legs de cet auteur. Rien d'étonnant à ce que Selvon lui-même ait choisi des métaphores tirées du domaine musical pour décrire sa pratique d'écriture :

I started [to write The Lonely Londoners] in straight English and then I slid into dialect because the dialect just slid of its own chord. When I thought about this description, it didn't come into the form of dialect, it came as straight English, as I write it down. And then the dialect just took over. And I think it makes a very nice blend of one isn't very conscious of. (Selvon 1973)

" Glisser », " moduler », " accorder » trouver la tonalité adaptée au propos, passer d'un registre à l'autre de façon quasi imperceptible et inconsciente, bref éviter les décalages abrupts, les transitions visibles, telle est, du moins dans la narration, la stratégie adoptée. Selvon a donc suivi la même logique dans le choix des marqueurs linguistiques que dans la répartition et les fonctions textuelles qu'il leur a assignées, une logique qui selon toute évidence relève plus de la modulation que de la juxtaposition et qui débouche sur un récit dans lequel, comme le concluait Maximin (1996, p. 363), "le fondu prédomine " et semble même de rigueur.

\section{4 $\mathrm{Du}$ dialecte dans le roman au roman en dialecte?}

Loin d'être paradoxal, ce contraste entre les juxtapositions de codes dans les dialogues et leur absence dans la narration vient réaffirmer l'opposition entre le monde dans lequel s'ancre le narrateur et le reste de la société. Aussi, les brouillages énonciatifs ne sont-ils pas le signe d'une complète polyphonie. Si les voix des «Lonely Londoners» se fondent à celle du narrateur, s'il est parfois difficile de savoir qui parle, en revanche celles des autres personnages, périphériques et non émigrés, sont clairement délimitées. Ainsi, le roman procède non pas à une déhiérarchisation des paliers discursifs mais plutôt à un réagencement à la fois des structures discursives caractéristiques du récit romanesque et des normes tacites d'inscription des parlers "illégitimes ». Ici, les modulations ne sont pas déclenchées par le

17 "The Lonely Londoners : Sam Selvon and the Literary Heritage », journee de conférences tenues au Musée de Londres, le 7 octobre 2000. 
passage d'un palier discursif à l'autre mais par celui d'un groupe de locuteurs (comprenant le narrateur) à l'autre. Dans un même texte, l'auteur parvient donc à représenter (dans les dialogues) puis à subvertir (dans la narration) les rapports de force sociolinguistiques hors-textuels. Ce faisant, il ébranle aussi les fondements de la distinction entre la "littérature en dialecte" regroupant des textes intégralement écrits en dialectes et le "dialecte en littérature", regroupant les textes dans lesquels le dialecte est en contraste avec d'autres registres. Selon Carpentier (1990), seul le second cas de figure impose une traduction dans un dialecte cible, inventé ou réel. The Lonely Londoners est narré dans un style dialectal, il met en scène des personnages qui s'expriment eux aussi en dialecte; "I consider The Lonely Londoners to be my first full length dialect novel " clamait souvent son auteur... Et pourtant les rares bribes de conversation émanant de personnages périphériques, les quelques répliques isolées délibérément représentées en anglais standard nous transportent dans la seconde catégorie dans laquelle la recréation du dialecte s'impose.

Loin de les évacuer, Selvon réintègre les langue et tradition littéraires anglaises, mais il le fait par la bande. S'il accorde à l'anglais littéraire «standard " une place bien marginale, le narrateur n'hésite toutefois pas à s'inspirer de la prose d'un Dickens, voire d'un T. S. Eliot (Brown 1996), à utiliser, à l'occasion, des structures syntaxiques ou un vocabulaire en dehors du " dialecte ". Ceci lui permet d'ailleurs aussi de contourner l'un des problèmes souvent rencontrés par les auteurs écrivant uniquement en créole: celui de l'expression de concepts abstraits et de descriptions ${ }^{18}$.

Au terme de cette analyse, le caractère motivé des stratégies microtextuelles de créolisation et "cette ingéniosité linguistique " qu'évoquait Ramchand apparaissent plus clairement. Chaque marqueur semble en effet avoir été consciencieusement choisi de façon à satisfaire critères de lisibilité et visée expressive. Chaque indice entrant dans le système sémiotique de l'œuvre trouve sa place et sa justification selon des critères bien plus variés et précis que ceux d'un simple réalisme ou de contraintes extérieures: l'usage du you polysémique ainsi que la forme de base à tous les temps et personnes

${ }^{18}$ Ce problème est soulevé et analysé par Hazaël-Massieux $(1993 ; 1996)$ et Raphaël Confiant (dans Ludwig 1994, pp. 171-180). 
favorisent des brouillages énonciatifs et temporels délibérés qui ont un impact direct sur les structures discursives et narratives du roman. Au delà des questions de lisibilité, l'absence d'eye dialect permettait aussi de passer plus doucement, dans la narration, du registre descriptif à l'anecdote. De même, les créolismes lexicaux ne semblent pas avoir été choisis au hasard: ce sont essentiellement les termes tirés du champ lexical de la parole, un thème central du roman, qui sont mobilisés. La question qui se pose alors est la suivante : comment la traduction qui bouleverse l'œuvre dans sa matérialité même peut-elle recréer les fonctions qu'assumait cette dernière ?

\section{Traduire The Lonely Londoners}

Ce roman écrit presque totalement en dialecte impose, en raison même de ce petit "presque", une traduction qui soit elle aussi en dialecte. Comment recréer les contrastes et cette tonalité constitutives du texte original ? Par quel dialecte d'arrivée substituer le dialecte de départ ? Existe-t-il un dialecte cible approprié ? Sinon, peut-on en inventer un ?

\subsection{Un équivalent à construire}

Pour répondre à ces questions, on se tournera vers les Antilles françaises, toutes désignées en raison de leur proximité géographique mais surtout de leur richesse linguistique. Cette démarche se heurte à un premier obstacle à la fois formel et politique. Tandis que Selvon représenta une variété mésolectale, accessible aux lecteurs noncréolophones, les créoles antillais à base lexicale française ont une syntaxe radicalement différente de celle du français. Du côté des Petites Antilles, ces créoles ont été en outre codifiés selon une graphie largement phonétique instaurant une déviance minimale sans laquelle, selon le linguiste Pinalie, " le créole ne serait jamais sorti de son statut de langue minorée et de sous-produit de la langue standard » (2000, p. 46). En somme, la codification du créole écrit a été conçue de façon à ce que ce dernier acquière le statut de langue distincte, de façon à ce que créole et français soient mutuellement inintelligibles. Derrière les différences formelles et linguistiques entre créoles à base lexicale anglaise et française se profilent donc aussi des enjeux politiques distincts. D'un point de vue traductionnel, ceci a deux conséquences : 
1) si l'on accepte le principe de "lisibilité symétrique " ${ }^{19}$ comme premier critère de traduction, il faudra recréer de toutes pièces un " français vernaculaire créolisé $~^{20} ; 2$ ) ce faisant on s'inscrit en quelque sorte à contre-courant du projet politico-linguistique des élites (linguistes et romanciers) des Antilles françaises qui cherchent au contraire à renforcer l'opacité du créole par rapport au français.

Dans ces conditions, faut-il opter pour une traduction en créole (basilectal) ? Faut-il renoncer à créoliser ? Faut-il s'inspirer d'un français régional martiniquais ou haitien ? Jusqu'à quel point peut-on s'inspirer du français créolisé des personnages romanesques de Chamoiseau, Confiant, Pépin ou Pineau ? Contrairement à ce que suggérait récemment Degras (2000), il semble qu'en matière de créolisation, le traducteur francophone, loin d'être dépourvu de ressources, n'ait en fait que l'embarras du choix. Comment choisir? Toute sélection suppose l'établissement d'une équivalence structurale du type : « l'anglais créolisé représenté dans The Lonely Londoners est au lecteur source ce que tel français créolisé est au lecteur cible » et ce, même dans le projet de traduction le plus littéral, le plus foreignizing qui soit. Car, comme le précise le tenant du foreignizing lui-même : " the key issue is not simply a discursive strategy (fluent or resistant) but always its intention and effect » (Venuti 1998, p. 188). En somme, l'idée que l'on se fait du lecteur de l'original et de celui de la traduction conditionne toujours le choix. J'adopte ici la position de Lane-Mercier, selon qui :

Prise [...] dans un processus politique implicite ou explicite, la traduction des sociolectes littéraires n'est ni une démarche vouée à l'échec (Berman) ni une démarche qui soulève le seul problème des équivalences (Folkart). C'est une démarche qui, s'embrayant sur le projet esthético-idéologique du texte source pour s'intégrer à celui du texte cible, vise des fins précises en créant un lecteur apte à les saisir. (Lane-Mercier 1995, p. 87)

${ }^{19}$ J'emprunte le concept à Vidal (1994, p. 189).

${ }^{20}$ Le projet du GERB (Université de Bordeaux) illustre cette nécessité et offre une démarche originale pour inventer un équivalent traductionnel au créole jamaïcain représenté dans les nouvelles d'Olive Senior (cf. Raguet-Bouvard 2000). 
Le concept de lecteur modèle développé par Eco (1979) vaut peut-être la peine d'être mobilisé. En effet, défini comme un ensemble de stratégies, d'indices textuels (Eco 1979, p. 75) et de conditions de succès remplies (ou non) à travers la lecture du roman, ce concept qui propose donc une construction du lecteur à partir d'éléments textuels a l'avantage de saboter l'opposition traductologique entre équivalences formelle et dynamique, opposition sur laquelle s'embrayent les clivages du type traduction sourcière ou cibliste, littérale ou hypertextuelle, etc. Tout texte, original ou traduction, prévoit en quelque sorte son lecteur. Aussi, avant de souscrire à telle ou telle approche traductionnelle, interrogeons quel serait, dans The Lonely Londoners, l'image de ce lecteur.

Lorsqu'on rassemble les indices identifiés au fil de cet essai, on découvre, selon moi, dans le roman de Selvon, un projet assez clair : celui de représenter et de réhabiliter un code linguistique mais aussi une communauté caractérisée tant par son rang social que par ses origines culturelles. Là émerge un premier problème de traduction : si la recherche de marqueurs diastratiques est relativement aisée, le français étant bien doté en expressions populaires (Sanders 1996, p. 43), en revanche rares sont les romans dans lesquels ce français populaire sort du domaine dialogal, et encore plus rares sont ceux dont le narrateur s'exprime dans un registre essentiellement vernaculaire. Cet aspect est d'autant plus problématique que les normes et conventions du bien écrire se font plus strictes en matière de littérature traduite. Au cœur du projet de Selvon réside donc le désir évident d'établir un dialogue avec le lecteur non-créolophone et non-caribéen, d'amener ce lecteur à découvrir ces personnages et leur langue de telle façon que soient révisées les préconceptions qu'il pouvait en avoir. $\mathrm{Si}$ elle demeure tout à fait subjective (mais laquelle ne l'est pas ?), cette lecture souligne l'importance d'avoir opté pour une représentation radicalement nouvelle qui ne charrie pas avec elle les connotations et les présupposés encadrant traditionnellement l'inscription romanesque du créole. Cette lecture permet également de saisir pourquoi l'auteur a adopté le vernaculaire comme langue de narration et personnalisé la parole de ses personnages : "Si vous croyiez qu'on parle tous pareil, détrompez-vous " semble nous dire le narrateur... à différents personnages et différentes situations correspondent différents codes et performances, ce qui n'empêche pas l'établissement, au niveau macrotextuel, d'une logique structurale, de réseaux d'alliance (narrateur 
solidaire des Lonely Londoners), d'oppositions (Harris vs. autres " lonely londoners »; narrateur+Lonely Londoners vs. personnages non-émigrés) et d'associations (récits plus créolisés, descriptions lyriques plus " standard »). Enfin et surtout, l'analyse du texte suggère à quel point il était primordial de tout mettre en œuvre pour rejoindre le lecteur non-caribéen. En ce sens, traduire The Lonely Londoners dans un créole basilectal inaccessible au lecteur non-créolophone reviendrait selon moi à nier le projet inscrit dans le texte de Selvon. Là réside peutêtre l'obstacle traductionnel le plus difficilement surmontable. Quel que soit son statut hors-textuel, la langue des « lonely Londoners » est représentée dans un anglais créolisé ou un créole très anglicisé mais en tous les cas dans un code lisible, et dans une large mesure pensé pour le lecteur non-caribéen. Cet anglais créolisé, suggère le roman, devrait avoir sa place dans le champ des littératures d'expression anglaise, tout comme les « lonely Londoners " devraient avoir la leur dans la capitale britannique. Ce glissement métaphorique de la ville à la parole, de l'espace physique à l'espace discursif, pourrait sembler douteux s'il n'était inscrit dans le texte même. En effet, tandis que le narrateur évoque au début du roman l'éventualité d'un retour de Moses à Trinidad, un retour «aux sources» que ce personnage émigré depuis une dizaine d'années envisage à plusieurs reprises, le roman se clôt sur une tout autre perspective :

Daniel was telling him [Moses] how over in France all kinds of fellars writing books what turning out to be best-sellers. Taxi-driver, porter, road-sweeper - it didn't matter. One day you sweating in the factory and the next day all the newspapers have your name and photo, saying how you are a new literary giant.

He watch a tugboat on the Thames, wondering if he could ever write a book like that, what everybody would buy. (TLL, p. 126) (je souligne)

Dans sa facture même, The Lonely Londoners s'offre en fait comme une réponse à l'ultime question de Moïse : il est bien possible d'écrire un livre comme ça.

D'un point de vue traductionnel, l'enjeu serait donc de parvenir à recréer les fonctions structurales, identificatrices et poétiques du dialecte source mais également, dans la mesure du possible, ses fonctions narratives. En ce sens, formes et fonctions deviennent difficilement dissociables. On ne peut traduire les secondes sans 
prendre en considération les premières. Si la traduction littérale est difficilement envisageable, il est par contre possible d'employer non pas des marqueurs mais des stratégies analogues à celles adoptées par l'auteur. Ici, il s'agirait par exemple d'éviter, comme l'a fait Selvon, les formes déjà stéréotypées - rejeter l'eye dialect, les ellipses et apostrophes, sans parler de la traditionnelle élision du $r$-; restreindre les créolismes lexicaux opaques aux termes clés; cultiver au contraire les particularités prosodiques; En matière syntaxique, il faudrait par exemple jouer sur l'usage des conjugaisons, les pronoms personnels. Enfin, dans la mesure où le texte de départ est écrit dans un registre essentiellement vernaculaire, on choisira de préférence, entre deux tournures de lisibilité équivalente, celle qui s'apparente le plus au vernaculaire créole à base lexicale française. On écrira messieurs et dames plutôt que Mesdames et Messieurs; on traduira job ou work par travail ou job plutôt que par boulot; trouble par tracas plutôt que pétrin etc.

Cette démarche qui consiste à rechercher non pas un dialecte d'arrivée mais des options microtextuelles n'est toutefois pas sans danger. Le problème n'en est pas un d'équivalent ni d'authenticité mais de légitimité. En effet, comme je l'ai déjà évoqué, ce type de créolisation vernaculaire contredit les préceptes des penseurs, linguistes et romanciers des Antilles françaises les plus en vue; elle néglige par exemple les recommandations d'un Glissant selon qui :

II nous faut opacifier le créole par rapport au français ou déstructurer le français par rapport au créole pour pouvoir maîtriser les deux, pour pouvoir sortir du "petit nègre". Il faut donc bien constituer l'originalité du créole par rapport au français et l'originalité du français par rapport au créole (la créolisation n'est en rien un méli-mélo). (Glissant 1996, pp. 52-53)

Ou bien celles des auteurs de l'Éloge de la créolité qui prônent l'usage littéraire du créole, et la créolisation littéraire, sans manquer de mettre en garde leurs lecteurs contre un usage "irresponsable " de l'interlecte : « dépositaire d'un génie multiple, l'interlecte peut bien, si l'on n'y prend garde, être le fossoyeur pur et simple du génie. Chaque fois qu'il nous dispense du travail critique de l'écriture, l'interlecte (serviteur attentionné et omniprésent) constitue le danger d'une aliénation subreptice mais terriblement efficace ". (Bernabé et al., 1989 , p. 49). Le lourd héritage du «français-banane » et la volonté 
d'opacifier le créole par rapport au français vont donc de pair et donnent lieu à une méfiance justifiée envers toute représentation d'un " français créolisé vernaculaire ». Comment recréer les fonctions du dialecte cible tout en tenant compte du contexte politico-linguistique prévalant dans les Antilles françaises?

Une solution possible consisterait à reléguer au second plan un critère qui a traditionnellement prévalu sur le reste: le principe d'identification, principe qui non seulement légitime mais appelle le stéréotype. Il ne s'agit bien évidemment pas de faire fi de tout réalisme mais, contrairement à ce que proposait Vidal (1994) dans la traduction du vernaculaire noir américain, de rejeter «les marqueurs de la Négritude les plus suggestifs " (Vidal 1994, p. 191) au profit de particularités lexicales et syntaxiques puisées dans le français oral des Antilles mais encore relativement peu exploitées dans le littéraire. Il s'agit en somme de recréer l'altérité des voix des "Lonely Londoners" et de recréer les liens de connivence et de distanciation présents dans l'original, sans capitaliser sur les représentations existantes et donc déjà stéréotypées du personnage noir émigré. Cette démarche est ici envisageable dans la mesure où le dialecte constitue la principale langue du récit (l'association entre le code et l'identité culturelle se fera donc de toute façon à l'échelle du roman). Elle parâ̂t légitime dans la mesure où elle repose sur un travail de création à partir du créole et du français plutôt que sur l'exploitation de représentations toutes faites. Enfin, elle semble appropriée à l'écriture de Selvon, une écriture qui relevait elle-même d'une expérimentation conduite selon des critères largement plus vastes et plus complexes que l'effet de réel. La démarche traductionnelle établie, reste à présent à sélectionner les ressources.

\subsection{Les ressources littéraires}

Si, comme l'a montré Lavoie (1997), il est possible de trouver dans les romans antillais d'expression française des options pour la traduction du vernaculaire noir américain, il semble a fortiori approprié de commencer par explorer ces romans. Les stratégies de créolisation dans les romans franco-antillais, en particulier ceux issus de l'école de la Créolité (Chamoiseau, Confiant, Pépin, Pineau) ont d'ailleurs déjà fait l'objet de plusieurs études (Deltel 1992; Hazaël-Massieux 1988, 1989, 1993; Prat 1992; Jermann 1994; De Souza 1995; N'Zengou-Tayo 
1996). De ces travaux et de ma propre lecture des romans émergent quelques constats. Tout d'abord, le lexique constitue, de loin, le lieu où l'inscription du créole est la plus manifeste. En comparaison, la syntaxe semble relativement peu sollicitée. Les juxtapositions français/créole et donc les traductions sont fréquentes. Enfin et surtout il semble que les narrateurs de Chamoiseau, tout en affichant leur antillanité, n'en oublient pas pour autant les règles du bien écrire. Dans leur manifeste, les tenants de la Créolité enjoignaient le romancier créole à utiliser pleinement sa langue vernaculaire mais également à démontrer que la société antillaise avait conquis le français. Contrairement à l'auteur de The Lonely Londoners, les romanciers se réclamant de cette école $s$ 'inspirent des registres les plus vernaculaires (représentés en graphie créole) mais aussi des plus châtiés, cultivant même les préciosités et les pédantismes dans une visée ironique (Prat 1992).

On peut alors se demander, avec Hazaël-Massieux, si dans ces romans, l'accession de la créolité à la narration ne traduit pas moins un allégement des normes d'écriture quant à la place textuelle des vernaculaires, que le passage « du créolisme honteux au créolisme noble " (Hazaël-Massieux 1996a, p. 678). En effet, le créolisme lexical apparaît désormais débarrassé de son stigma, accepté, voire cultivé, à tel point que les auteurs n'hésitent pas à " en rajouter ", selon une accumulation "plus vraie que nature» (Hazaël-Massieux 1996a, p. 679) en créant des textes de plus en plus opaques pour le lecteur peu familier avec les créoles ou français antillais (DeSouza 1995). Il en va par contre tout autrement du créolisme syntaxique qui semble, quant à lui, avoir quelque peine à se tailler une place dans le littéraire et $a$ fortiori dans la narration... Sans doute parce que la différence syntaxique peut trop facilement passer pour une déviance et donc pour une faute.

Des romans de la Créolité on recueillera donc plus de créolismes lexicaux qu'il n'en faut ainsi que de nombreux marqueurs prosodiques propres au français des Petites Antilles; on découvrira surtout l'illustration des règles du jeu qui sous-tendent la créolisation littéraire pratiquée par cette école, une créolisation qui s'avère au bout du compte plus sophistiquée que vernaculaire. Pour retrouver des exemples de créolisation plus proches du vernaculaire, il faut tout d'abord sortir du canon, consulter des romans moins reconnus et qui adoptent des stratégies différentes tels que ceux de Dracius-Pinalie 
( $L$ 'autre qui danse) $)^{21}$ ou bien encore les traductions françaises des romans créoles de Confiant moins connues des lecteurs francophones (ex : Chimères d'en-ville). Mais, il semble qu'il faille aussi et surtout sortir complètement du domaine littéraire, pour chercher des particularismes dont les écrivains se sont jusqu'à présent peu ou pas inspirés.

\subsection{Au delà du littéraire}

Comparativement au créole, le français régional des Antilles a jusqu'à présent fait l'objet de peu d'études. Toutefois, outre les dictionnaires existants, les travaux de Hazaël-Massieux sur le français martiniquais et ceux de Pompilus sur le français haïtien révèlent des particularismes syntaxiques susceptibles d'être exploités dans le littéraire. Par exemple, aux fluctuations entre l'emploi de l'accusatif et du nominatif pour les pronoms compléments dans The Lonely Londoners pourrait correspondre, dans la traduction, l'incertitude sur l'usage des pronoms $l e$ et $l u i$, un phénomène fréquent mais non exclusif aux Antilles. L'absence occasionnelle d'accord en genre et en nombre du substantif que l'on trouve dans le texte de Selvon se rencontre parfois aussi dans les français antillais (Hazaël-Massieux 1996a; Telchid 1997). En anglais comme en français créolisés le déterminant est bien souvent facultatif dans des contextes autres que proverbiaux (Telchid 1997), phénomène là encore représenté dans The Lonely Londoners. De même, d'après Pompilus, le français haîtien admet l'utilisation du présent à valeur de passé :

La forme verbale non marquée peut dénoter dans l'idiome maternel [le créole] à la fois un intemporel, un présent d'habitude et un présent lié au passé. Cette forme non marquée est couramment transposée par les locuteurs moyens en un présent français là où seul le passé composé peut convenir. (Pompilus 1979, p. 132)

Ce particularisme permettrait donc peut-être de recréer dans la mesure du possible, les ambiguittés temporelles présentes dans l'original. Au delà de la morphosyntaxe, le procédé de topicalisation (par exemple : beau qu'il est beau; c'est viens que je viens) pourrait traduire, tant du point de vue de la forme que du sens, celui de fronting. Les marqueurs lexicaux, moins fréquents et plus faciles à traduire, ont également des

${ }^{21}$ Je remercie Katia Merine d'avoir attiré mon attention sur cette auteure. 
équivalents en français créolisé. Ainsi la conversion de certains adjectifs en adverbes (ex. : bad pour badly) caractérise aussi le français antillais (ex.: Il te regarde fixe Telchid 1997; il faut parler franc Pompilus 1979).

Enfin, en dépit des contraintes de lisibilité, il faut se garder de rejeter le créole, en particulier le créole martiniquais. En effet, comme on l'a évoqué plus haut (cf. note 9) ce dernier fut, au XIX ${ }^{\mathrm{e}}$ siècle, l'une des principales, sinon la première langue vernaculaire parlée à Trinidad. S'il n'est plus parlé aujourd'hui, hormis dans quelques rares villages de montagne, ce créole a laissé son empreinte sur celui qui lui a succédé : le vernaculaire créole anglais. Ainsi, outre l'influence syntaxique relevée par Solomon (1993), bien des images, des tournures et expressions contemporaines, présentes dans le texte de Selvon, ont un équivalent littéral en créole martiniquais. Par exemple, les tournures du type to talk a old talk, to smile a big smile, fréquentes dans le texte original ont un équivalent littéral en créole basilectal qui, moyennant l'ajustement graphique, serait accessible au lecteur non-créolophone : palé an vyè palé (ou parlé un vieux parlé). Enfin, sans pour autant traduire le texte en créole martiniquais, il convient de garder cette langue en tête non seulement parce que sa forme est assez proche de celle du créole trinidadien, mais aussi et surtout pour éviter ces «connotations indésirables » résultant de l'emploi de tournures ou lexèmes trop associés, pour le lecteur antillais, à un français « métropolitain ».

Le but de cette démarche n'est évidemment pas d'établir une liste exhaustive, ni de remplacer systématiquement et bêtement un même phénomène linguistique par son équivalent formel, mais plutôt de puiser dans les représentations littéraires et dans les particularités extra-littéraires, afin de recréer, selon une logique de compensation (Carpentier 1990) et non de calque, une parole créolisée qui tienne compte à la fois des fonctions narratives et poétiques de l'original, du principe de lisibilité symétrique, enfin et surtout, des normes d'acceptabilité et des enjeux qui sous-tendent la représentation du créole et des esthétiques créolisées dans le contexte francophone. 


\section{Conclusion}

À travers l'étude de la représentation du «modified Trinidadian dialect " de Selvon, cet essai a tenté de démontrer que l'enjeu de la traduction de The Lonely Londoners était peut-être moins de parvenir à recréer une représentation " authentique » de ce sociolecte romanesque ou de décider si l'on va opter pour une stratégie de résistance ou de transparence, qu'à concilier le projet esthético-idéologique de départ aux impératifs de lisibilité mais aussi et surtout aux « règles du jeu » de créolisation littéraire résultant du projet politico-linguistique de revalorisation du créole dans la sphère francophone.

En somme, la traduction de The Lonely Londoners est complexe et enrichissante précisément parce qu'elle nous oblige à sortir des dichotomies traditionnelles et à penser la traduction comme un processus tripartite entre langues-cultures française-anglaiseantillaise. Loin de substituer une dialectique étrangère (AngleterreCaraïbe) par une dialectique domestique (France-Antilles), ou de remodeler l'étranger à traduire en fonction des termes du débat domestique (ce qui contribuerait à raffermir chacune des positions en optant par exemple pour une traduction en créole basilectal ou en argot parisien), la traduction vient en quelque sorte perturber ce débat: elle déstabilise à la fois les normes d'acceptabilité "traditionnelles", françaises, mais aussi les « règles du jeu » de la créolisation littéraire définies par les élites des Antilles françaises telles que Bernabé et al. ou Glissant. L'institution littéraire française est-elle prête à ouvrir ses portes à des narrateurs tels que celui de The Lonely Londoners? Dans les Antilles françaises, est-on prêt à " désopacifier » le créole ? Si d'un côté, il semble s'être établi un canon d'œuvres francophones créolisées au sein duquel The Lonely Londoners n'a pas tout à fait sa place, dans les Antilles anglophones, l'écriture de Selvon en a inspiré plus d'un. Les romans du Canadien Austin Clarke (A Bigger Light 1975), ceux du Trinidadien Earl Lovelace (The Dragon Can't Dance 1979; The Wine of Astonishment 1982, Salt 1996) du Guyanais David Dabydeen (The Counting House 1996) ou de la Jamaïcaine Patricia Powell (Me Dying Trial 1993, A Small Gathering of Bones 1994) pour n'en citer que quelques-uns en sont la preuve tangible. Optant pour des stratégies de créolisation qui s'apparentent à celles mises au point par Selvon, ils expriment mieux que n'importe quelle formule d'usage l'influence que l'œuvre de cet auteur continue d'exercer sur les "nouvelles» 
générations d'écrivains. Bien qu'elles se comptent encore sur les doigts d'une seule main, les rares traductions en français de ces romans réalisées par Hélène Devaux et Ananda Devi ${ }^{22}$, des traductions dans lesquelles apparaissent, sous une forme encore ponctuelle et à petite échelle, des modes de créolisation syntaxique originaux, suggèrent pour leur part qu'il existe peut-être, si minime soit-il, un " espace de jeu traductionnel " pour de telles œuvres. Ces exemples laissent-ils entendre qu'en matière de créolisation, la littérature traduite serait porteuse d'innovation? Les cas sont encore trop peu nombreux pour que l'on dresse de telles conclusions. Toutefois, une chose est certaine : à l'heure où, dans cette même francophonie, on s'interroge sur les risques d'érosion (Degras 1996; Hazaël-Massieux 1996b; Bonnet 1998), d'exotisme (Jorif 1990), voire de " terrorisme culturel » (Condé in Pfaff 1993, p. 165) que comporterait une pratique trop restrictive et systématique de l'esthétique prônée par Bernabé et al (1989), traduire Selvon et ses successeurs contribuerait sans aucun doute à donner à cette expression littéraire du métissage, un visage différent.

\section{Université McGill}

Je tiens à remercier Judith Lavoie d'avoir lu et commenté les versions préliminaires de cet essai. $\mathrm{La}$ recherche dans laquelle s'inscrit la présente étude est financée par le Conseil de recherche en sciences humaines du Canada.

\section{Références}

AKAI, Joanne (1997). "Creole... English: West Indian Writing as Translation ». TTR, vol. X, $\mathrm{n}^{\circ} 2$, pp. 165-196.

ASHCROFT, Bill Gareth GRIFFITH et Helen TIFFIN (1989). The Empire Writes Back: Theory and Practice in Post-Colonial literatures. London, Routledge.

ALLSOPP, Richard (ed.) (1996). Dictionary of Caribbean English

22 Hélène Devaux a traduit The Dragon can't Dance (La danse du dragon, Éditions le Monde Noir Poche, 1979; éditions du Serpent à Plume, 1999). C'est également elle qui a traduit Moses Ascending de Selvon (Éditions Caribéennes, 1987); Ananda Devi a traduit The Counting House (Terres maudites, Éditions Dapper, 2000). 
Usage. Oxford, Oxford University Press.

BANDIA, Paul (1996). "Code-switching and code-mixing in African creative writing: some insights for translation studies ". TTR, vol. IX, $\mathrm{n}^{\circ} 1$, pp. 139-153.

BARRATT, Harold (1988). "From Colony to Colony: Selvon's Expatriate West Indians ». Nasta (ed.), Critical Perspectives on Sam Selvon. pp. 250-259.

BERMAN, Antoine (1984). L'épreuve de l'étranger. Culture et traduction dans l'Allemagne romantique. Paris, Gallimard.

BERNABÉ, Jean (1992-1993). « De la négritude à la créolité : éléments pour une approche comparée ». Études françaises. vol. $38, \mathrm{n}^{\circ}$ 2/3, pp. 23-38.

BERNABÉ, Jean, Raphaël CONFIANT et Patrick CHAMOISEAU (1989). Éloge de la créolité. Paris, Gallimard.

BONNET, Véronique (2000). "L'âme prêtée à l'écriture ». Notre librairie. ${ }^{\text {os }}$ 138-139, pp. 91-97.

- (1998). "La voix du petit peuple ». Notre librairie. $\mathrm{n}^{\circ} 135, \mathrm{pp}$. 108-111.

BOURDIEU, Pierre (1982). Ce que parler veut dire. Paris, Fayard.

BRERETON, Bridget (1981). A History of Modern Trinidad 17831962. Kingston-Port of Spain-London, Heinemann.

BRODSKY, Françoise (1996). «La traduction du vernaculaire noir : l'exemple de Zora Nearle Hurston ». TTR, vol. IX, $\mathrm{n}^{\circ}$ 2, pp. 165-178.

BROWN, Wayne (1996). "A Greatness and a Vastness: The Search for God in the Fiction of Sam Selvon ». ARIEL, vol. 27, $\mathrm{n}^{\circ} 2$, pp. 3546.

CARPENTIER, Godeleine (1990). "Traduire la forme, traduire la fonction : la représentation du dialecte dans deux genres littéraires, le 
roman et la poésie ». Ballard (éd.), La traduction plurielle. Lille, Presses universitaires de Lille.

CHAUDENSON, Robert (1979). Les créoles français. Evreux, Nathan.

CHUKWU, Augustine Emmanuel (1984). Home and Exile: A Study of the Fiction of Samuel Selvon (West Indies). Ph.D., Universite du Nouveau-Brunswick.

ClARKE, Austin (1994). A Passage Back Home: a personal Reminiscence of Sam Selvon. Toronto, Exile Editions.

COLLINS, Merle (1998). "Writing and Creole Language Politics: Voice and Story ». Balutansky and Sourieau (ed.), Caribbean Creolization, pp. 89-95.

CONFIANT, Raphaël (2000). "Traduire la littérature en situation de diglossie ». Palimpsestes, $\mathrm{n}^{\circ}$ 12, pp. 49-60.

D'COSTA, Jean (1983). « The West Indian Novelist and Language: a Search for a Literary Medium ». D. Carrington (ed.), Studies in Caribbean Language. Society for Caribbean Linguistics, St. Augustine, Trinidad, pp. 252-265.

DEGRAS, Priska (2000). « Spécificité de l'oralité caraïbe : quels choix de traduction ». Palimpsestes, ${ }^{\circ} 12$, pp. 39-48.

- (1996). «La littérature caraïbe francophone : esthétiques créoles ». Notre librairie, $\mathrm{n}^{\circ} 127$, pp. 6-16.

DELTEL, Danielle (1992). "La créativité du créole dans le roman de langue française : Patrick Chamoiseau et Axel Gauvin ». Convergences et divergences dans les littératures francophones. Paris, L'Harmattan, pp. 182-200.

DE SOUZA, Pascale (1995). «De la citation à la créolisation». CONDÉ Maryse et Madeleine COTTENET-HAGE (dir.), Penser la créolité. Paris, Karthala pp. 173-190. 
DICKISON, Swift (1994). Transnational Carnival an Creolised garden: Caribbean Cultural Identity and Rooting in the narratives of Sam Selvon and Merle Hodge. Ph.D., Washington State University.

DONNELL, Alison and Sarah LAWSON WELSH, (eds.) (1996). The Routledge Reader in Caribbean Literature. London, Routledge.

ECO, Umberto (1979). Lector in fabula, le rôle du lecteur. Paris, Livre de Poche.

FABRE, Michel (1982). " Moses and the Queen's English: Dialect and Narrative Voice in Samuel Selvon's London Novels ». Nasta (ed.), Critical Perspectives on Sam Selvon. Washington, Three Continents Press, pp. 213-222.

- ([1979] 1988). « From Trinidad to London: Tone and Language in Samuel Selvon's Novels ».

GLISSANT, Édouard (1996). Introduction à une poétique du divers. Paris, Gallimard.

HAZAËL-MASSIEUX, Guy et Marie Christine (1996a). "Quel français parle-t-on aux Antilles ». Robillard D. de et M. Beniamino, (éds), Le français dans l'espace francophone, vol. 2. Paris, Champion, pp. 665-680.

HAZAËL-MASSIEUX, Marie-Christine (1996b). « Les avatars de la littérature en créole ». Notre librairie, ${ }^{\circ} 127, \mathrm{pp}$. 18-28.

-(1993). Écrire en créole: oralité et écriture aux Antilles. Paris, L'Harmattan.

- (1989). «Solibo magnifique, le roman de la parole ». Antilla, spécial $\mathrm{n}^{\circ} 11, \mathrm{pp} .32-36$.

- (1988). " À propos de Chronique des sept Misères : une littérature en français régional pour les Antilles ». Études créoles, vol. XI, $\mathrm{n}^{\circ} 1$, pp. 118-131. 
HILL, Errol (1972). The Trinidad Carnival: Mandate for a National Theatre. Austin, University of Texas Press.

JERMANN, Alexandra (1994). "Les traditions créoles dans la littérature contemporaine de la Guadeloupe et de la Martinique». Glaser and Paush (éds.), Caribbean Writers, Between Orality and Writing. pp. 93-110.

JONASSAINT, Jean (1992-93). "Des conflits langagiers dans quelques romans haïtiens ». Études françaises, vol. 28, n²/3, pp. 3948 .

JORIF, Richard (1990). Les persistants lilas. Paris, Gallimard.

JOSEPH, Margaret-Paul (1992). Caliban in Exile: The outsider in Caribbean Fiction. New York, Greenwood.

LANE-MERCIER, Gillian (1995). " La traduction des discours directs romanesques comme stratégie d'orientation des effets de lecture $»$. Palimpsestes, $n^{\circ}$ 9, pp. 75-91.

- (1989). La parole romanesque. Ottawa, Presses de l'Université d'Ottawa.

LAVOIE, Judith (1997). "Le français créolisé comme option de traduction du vernaculaire noir américain ». Présence francophone, $\mathrm{n}^{\circ}$ 51, pp. 117-138.

LINTVELT, JAPP (1978). "Modèle discursif du récit encadré ". Poétique, $\mathrm{n}^{\circ} 35$, pp. 352-365.

LOOKER, Mark (1996). Atlantic Passages : History, Community and Language in the Fiction of Sam Selvon. New York, Peter Lang, Studies of World Literature in English.

LOWRY WEIR, Ann (1982). "Style Range in the New English Literatures ", dans The Other Tongue: English across Cultures. Urbana, University of Illinois Press, pp. 307-322. 
LUC-CAYOL, Agnès (1986). Comportement des Antillais dans leur milieu d'origine et à Londres à travers quatre romans de Samuel Selvon: A Brighter Sun, Turn Again Tiger, The Lonely Londoners, Moses Ascending. Doctorat de $3^{\text {ème }}$ cycle, Université de Bourgogne, Dijon.

LUDWIG Ralph, (éd.) (1994). Écrire la parole de nuit. Paris, Gallimard, collection « Folio ».

MAIR, Christian (1990). «Naipaul's Miguel Street and Selvon's The Lonely Londoners - two Approaches to the Use of Caribbean Creole in Fiction ". The Journal of Commonwealth Literature, vol. 24, $\mathrm{n}^{\circ} 1$, pp. 138-154.

MAXIMIN, Colette (1996). Littératures caribéennes comparées. Pointe-à-Pître et Paris, Jasor et Karthala.

MICHELET, Isabelle (2000). " La situation sociolinguistique de la Martinique : enquête ». Mofwaz, $\mathrm{n}^{\circ}$ 5, pp. 71-80.

NASTA, Susheila and Anna RUTHERFORD, (ed.) (1995). Tiger's Triumph: Celebrating Sam Selvon. London, Dangarro Press.

NASTA, Susheila (1995). « Setting Up Home in a City of Words : Sam Selvon's London Novels ». Nasta Susheila and Anna Rutherford, (eds.), Tiger's Triumph: Celebrating Sam Selvon. London, Dangarro Press, pp. 78-95.

- (ed.) (1988). Critical Perspectives on Sam Selvon. Washington, Three Continents Press.

N'ZENGOU-TAYO, Marie-José (1996). "Littérature et diglossie: créer une langue métisse ou la "chamoisification" du français dans Texaco de Patrick Chamoiseau ». TTR, vol. IX, $\mathrm{n}^{\circ}$ 1, pp. 155-176.

OTTLEY, C. R. (1971). Creole talk (Trinibagianese) of Trinidad and Tobago. Port of Spain, Victory Printers.

PFAFF, Françoise (1993). Entretiens avec Maryse Condé. Paris, Kharthala. 
PINALIE, Pierre (2000). "Lecture et parole dans l'enseignement du créole à des non-créolophones ». Mofwaz, n ${ }^{\circ}$, pp. 161-172.

POMPILUS, Pradel (1979). « La langue française en Haiiti ». Valdman (éd.). Le français hors de France. Paris, Champion.

PRAT, Michel (1992). " Patrick Chamoiseau : Un émule de Gadda ? ". Maryse Condé (dir.), L'héritage de Caliban. Paris, Jasor, pp. 201-212.

RAMCHAND, Kenneth ([1982] 1988). "Song of Innocence, Song of Experience: Samuel Selvon's The Lonely Londoners as a Literary work». World Literature Written in English, vol. 21, pp. 644-654. (Réédité dans NASTA (éd.) 1988)

- (1970). The West Indian Novel and its Background. London, Faber and Faber.

REID, Vic (1949). New Day. New York, Alfred A. Knopff.

RICHARDSON, Maurice (1956). " New Novels ». The New Statesman and Nation. 29 décembre, vol. 52, p. 846.

ROBERTS, Peter (1988). West Indians and their Language. Cambridge, Cambridge University Press.

SANDERS, Carol (1996). 'Pourquoi qu'on dit des choses et pas d'autres?' Translating Queneau's français parlé in Zazie dans le métro and Le Chiendent. Palimpsestes, ${ }^{\circ} 10, \mathrm{pp} .41-48$.

SELVON, Sam (1998). The Lonely Londoners. London, Longman "Caribbean Writers Series », préfacé par Kenneth Ramchand, pp. 320.

- (1995). « Sam Selvon: Interview with Reed Dasenbrockand Feroza Jussawalla " Nasta and Rutherford, (ed.), Tiger's Triumph: Celebrating Sam Selvon. London, Dangarro Press, pp. 114-125.

-([1956] 1991). The Lonely Londoners. Toronto, TSAR. 
- (1987). L'ascension de Moïse. Trad. Hélène Devaux-Minie. Paris, Éditions Caribéennes.

-([1952] 1986). A Brighter Sun. London, Longman, Longman Caribbean Writers Series.

- ([1982] 1988). " Interview with Kenneth Ramchand ». Nasta, (ed.), Critical Perspectives on Sam Selvon. Washington, Three Continents Press, pp. 95-103.

- (1973). Entrevue avec Gerald Moore, "The English Novel Abroad - A programme in the Series». BBC Radio 3, enregistré le 26/11/1973 (National Sound Archive : AC MT 35716).

SOLOMON, Denis (1993). The Speech of Trinidad, A Reference Grammar. St Augustine (Trinidad), School of Continuing Studies of the Unversity of the West Indies.

TABUTEAU, Éric (1997). Images du multiculturalisme dans le roman antillais anglophone: Wilson Harris, George Lamming, V. S. Naipaul, Sam Selvon. Villeneuve d'Ascq, Presses Universitaires du Septentrion.

- (1993). "Love in Black and White: A Comparative Study of Samuel Selvon and Frantz Fanon ». Commonwealth Essays and Studies (CE\&S), vol. 16, $\mathrm{n}^{\circ} 2$, pp. 88-95.

TELCHID, Sylviane (1997). Dictionnaire du français régional des Antilles. Paris, Éditions Bonneton.

THIEME, John (1986). «The World Turns Upside Down: Carnival Patterns in The Lonely Londoners ». The Toronto South-Asian Review, $\mathrm{n}^{\circ}$ 5, pp. 191-204.

VENUTI, Lawrence (1998). The Scandals of Translation. Towards an Ethics of Difference. London, New York, Routledge.

VIDAL, Bernard (1994). « Le vernaculaire noir américain : ses enjeux pour la traduction envisagés à travers deux œuvres d'écrivaines noires, Zora Neale Hurston et Alice Walker ». TTR, vol. VII, $\mathrm{n}^{\circ} 2$, pp. 165204. 
WARNER, Keith Q. (1982). The Trinidad Calypso. London, Heinemann.

WINER, Lise (1993). Trinidad and Tobago. London, John Benjamins, " Varieties of English Around the World».

WYKE, Clement (1991). Sam Selvon's Dialectal Style and Fictional Strategy. Vancouver, University of British Columbia Press.

\section{RÉSUMÉ : The Lonely Londoners en français: l'épreuve du} métissage - En 1956, l'écrivain trinidadien Sam Selvon (1923-1994) publiait The Lonely Londoners, un roman rédigé intégralement dans une variété de langue jusque là stigmatisée et confinée au domaine dialogal: le vernaculaire créole trinidadien. Bien qu'il fasse aujourd'hui partie des «classiques " de la littérature anglo-caribéenne, ce roman n'a toujours pas été traduit en français. À partir d'une étude de la matérialité puis des fonctions textuelles de cette représentation, l'essai révèle que si The Lonely Londoners offre un exemple original de créolisation littéraire, sa traduction suppose quant à elle la représentation d'un français créolisé qui, en conflit apparent avec les normes du polysystème cible et les projets politico-linguistiques des élites franco-antillaises, n'a pas encore eu droit de cité dans le littéraire. Constatant l'existence d'équivalents micro-textuels aux marqueurs de créolisation présents dans l'original, l'auteure suggère que les enjeux sous-tendant la traduction d'un tel roman ne peuvent se définir en termes d'authenticité ou bien de choix entre une stratégie traductionnelle de résistance ou de transparence. En effet, s'inspirant à la fois des langues-cultures trinidadienne et britannique, The Lonely Londoners oblige à sortir des dichotomies traditionnelles et à penser la traduction comme un processus tripartite entre langues-cultures française-anglaise-antillaise. Loin de remplacer une dialectique étrangère (Angleterre-Caraïbes) par une dialectique domestique (France-Antilles), ou de remodeler l'étranger à traduire en fonction des termes du débat dans le contexte cible, la traduction vient en quelque sorte perturber ce débat et proposer de nouvelles avenues en matière de créolisation littéraire. 
ABSTRACT: Translating The Lonely Londoners into French : The Experience of Métissage - In 1956, The Trinidadian born writer Sam Selvon (1923-1994) published The Lonely Londoners, a novel entirely written in a language formerly stigmatised and whose literary representation was mostly confined to direct speech: Trinidadian Creole English. Although this novel has now become a classic of West Indian literature, it has not yet been translated into French. Based on an analysis of the materiality and the narrative functions of this literary dialect, the essay attempts to show that while The Lonely Londoners offers a unique example of text creolisation, its translation requires the recreation of a dialect that subverts the norms of acceptability of the French literary polysystem. Assessing the existence of micro-textual translation equivalents to the Creole forms used in the original, the author suggests that the translation problematics should not be addressed in terms of " authenticity" or even as a question of choice between one strategy or the other (fluency or resistance). Indeed, having himself bridged the gap between oral Creole and written British language-cultures, Selvon compels his translator to disregard traditional dichotomies in order to think translation as a three-part relationship between French, Caribbean (Creole), and English language-cultures. Far from replacing a foreign dialectics (Britain-West Indies) by a domestic dialectics (France-French Caribbean), far from modeling the foreign text according to the terms of the debate in the target context, translation may become a means for actually destabilising this very debate and proposing new approaches to literary creolisation.

Mots clés : Selvon, traduction, Trinidad, Créole, vernaculaire.

Key Words: Selvon, translation, Trinidad, Creole, vernacular.

Hélène Buzelin : Université McGill, Département de langue et littérature françaises, 3460 McTavish, Montréal (Québec), H3A 1X9;

Courriel : hbuzelin@hotmail.com. 